Elsevier required licence: (c) 2018. This manuscript version is made available under the CC-BY-NC-ND 4.0 license http://creativecommons.org/licenses/by-nc-nd/4.0/ 


\section{Investigation of some of the factors influencing fingermark detection}

Scott Chadwick ${ }^{1 *}$, Sebastien Moret ${ }^{1}$, Nilesh Jayashanka, ${ }^{1,2}$, Chris Lennard $^{3}$, Xanthe Spindler ${ }^{1}$, Claude Roux ${ }^{1}$

${ }^{1}$ University of Technology Sydney, Centre for Forensic Science, Broadway, 2007, Australia

2 Temasek Polytechnic School of Applied Science, 21 Tempines Avenue 1, 529757, Singapore

${ }^{3}$ School of Science and Health, Western Sydney University, Locked Bag 1797, Penrith, NSW, 2751, Australia

* Corresponding Author: Scott.Chadwick@uts.edu.au 


\section{Highlights}

- Over 14,000 fingermarks were developed and evaluated to investigate some of the factors that influence fingermark detection

- Fingermarks deposited on porous surfaces were more likely to be developed than fingermarks on non-porous surfaces

- Significant variability was observed between donors and within repeat depositions of the same donors fingermarks

- Reinforces the need for further research into the fundamentals of fingermark detection 


\section{Investigation of some of the factors influencing fingermark detection}

3 ABSTRACT:

4 The primary aims of fingermark detection research are to improve the quality and increase 5 the rate of detection of identifiable impressions. This is usually performed through the 6 development of new methods and technologies to provide alternatives to or improve current 7 procedures. While research of this nature is important to pursue, it fails to address the 8 underlying question related to the factors that affect the detection of a latent fingermark.

9 There has been significant research that has examined the differences between techniques, 10 donors and fingermark age, as well as the composition of latent fingermarks. However, they 11 tend not to focus on determining how these factors influence the quality of the developed 12 mark.

14 This study involved the development and evaluation of over 14,000 natural fingermarks 15 deposited on a variety of surfaces to examine the effect of substrate, age, donor variability 16 (both inter- and intra-), depletions and type of finger on fingermark development. 17 Fingermarks were deposited on four substrates (two non-porous and two porous) and 18 developed with either indanedione-zinc (IND-Zn) or cyanoacrylate followed by rhodamine $6 \mathrm{G}$ staining $(\mathrm{CA}+\mathrm{R} 6 \mathrm{G})$. Three independent assessors graded each mark on the quality of development using an absolute scale proposed by the UK Centre for Applied Science and Technology (CAST). The data generated from these assessments were then analysed for trends or other useful insights.

24 The results from this work reaffirm that individual substrate characteristics (and the choice of development technique) play a significant role in determining the number and quality of marks developed. It was found that fingermarks were more likely to be detected on porous substrates and to also be of a higher quality than on non-porous. The effect of fingermark donor variability was also explored, with significant differences observed between donors and within donors. This research shows that current detection techniques do not detect all available fingermarks, reinforcing the need for further research into the fundamentals of

31 fingermark detection in order to gain a better understanding of the techniques currently used. The study has identified considerations for the development of novel techniques and how we 
33 need to account for variability when designing fingermark research experiments.

Page $\mathbf{2}$ of $\mathbf{2 7}$ 
Page $\mathbf{3}$ of $\mathbf{2 7}$ 
36 Latent fingermarks, Indanedione-zinc, Cyanoacrylate, Detection

\section{Introduction}

40 Fingermark detection research has grown significantly over the last decade, expanding in 41 scope to incorporate a number of emerging technologies with the aim of improving the quality and quantity of fingermarks detected $[1,2]$. Examples of this include nanoparticlebased approaches, biochemical methods such as biomolecular recognition, and chemical imaging [3-6]. These new methods often adopt technologies that are theoretically more selective and sensitive to fingermark components than current methods. However, assessing the efficiency of both current and proposed new techniques is a difficult matter as fingermark composition and the factors that affect a fingermark deposit over time, as well as its detection, are not yet fully understood [7]. Therefore, before focusing on improving current techniques or investigating novel approaches, researchers in the field should endeavour to answer the following fundamental questions: (i) how many marks go undetected?; (ii) what factors affect the detection rate?; and (iii) what is the best way to assess a technique's detection efficiency?

These questions are far from being trivial and very few groups have made an attempt at evaluating the detection rate of any given techniques. This is further complicated in regard to our understanding of a 'detection rate'. Some groups focus on the effectiveness of the reaction to develop fingermarks, while others focus on the quality of the developed marks. While these two concepts are linked, research into new techniques generally focuses on the former and validation research on the latter. Focusing on one or the other will affect the reporting on the 'detection rate'. The most common claim in regard to the number of marks going undetected is from a paper by Jaber et al. where it was stated that 'over $50 \%$ escape detection of identifiable marks' [8]. This statement referenced a previous study that used a combination of controlled fingermarks and real exhibits (bank cheques), and compared the efficiency of indanedione-zinc (IND-Zn) and 1,8-diazafluoren-9-one (DFO). This study found that 219 identifiable fingermarks were developed on 150 out of 500 cheques with IND$\mathrm{Zn}$ [9]. While operational studies such as this can provide an informative snapshot of the expected effectiveness of a technique, it is unclear how this $50 \%$ value was calculated 
without knowing the total number of fingermarks originally deposited. There was also a difference in the number of marks recovered between the different techniques (IND-Zn recovered 50\% more marks than DFO). Additionally, if an assumption is made that at least one fingermark was deposited on each cheque then the detection rate is potentially lower, with only $30 \%$ of fingermarks being developed and, by extension, even less being of identifiable quality.

A broader examination of other large-scale fingermark studies (Table 1) reveals that the percentage of recovered fingermarks is incredibly variable. Unsurprisingly, the number of undetected marks is dependent on the detection method, the selection of substrates, the fingermark donors, and the time since deposition. Care must be taken when comparing results across studies and the values reported as there are generally variations (fingermark age, substrate, number of donors, technique formulation, development and environmental conditions, etc.) that may account for these differences. This brief discussion demonstrates that the answer to how many marks go undetected is not straightforward; many different parameters are involved. This is further illustrated by the study reported by Beaudoin et al. that showed that the number of marks detected is highly reliant on the substrate itself, with a range of $0.34-94 \%$ of marks undetected on different surfaces using the same detection technique [10].

Another factor that should be considered with these published results is that fingermarks were deposited with the conscious intent to leave an impression for the purposes of detection. It is also typical to annotate research specimens with a grid, outline or date to alert the researcher as to where the latent fingermarks are located, meaning that even very faint fingermarks are more conspicuous (or, at least, anticipated) than in genuine casework scenarios where weak fingermarks may be overlooked. It can be hypothesised that more realistic deposition scenarios - more in line with a pseudo-operational study [11] - would provide more accurate information on the efficiency of fingermark detection techniques. While these types of studies have been performed [12, 13], the number of marks undetected (and thus the percentage efficiency of the fingermark detection technique) cannot be calculated without knowing the initial number of marks deposited. This illustrates the complexity of assessing a technique's detection efficiency. 


\begin{tabular}{|c|c|c|c|c|c|c|}
\hline $\begin{array}{c}\text { Fingermark } \\
\text { Detection Technique }\end{array}$ & $\begin{array}{c}\text { Number and type of } \\
\text { Surfaces }\end{array}$ & $\begin{array}{l}\text { Number of } \\
\text { Donors }\end{array}$ & $\begin{array}{c}\text { Age of } \\
\text { Fingermarks }\end{array}$ & $\begin{array}{c}\text { Percentage of } \\
\text { marks not } \\
\text { detected* }\end{array}$ & $\begin{array}{c}\text { Percentage of } \\
\text { identifiable } \\
\text { marks* }\end{array}$ & Reference \\
\hline IND-Zn & 1 (White Copy Paper (WCP)) & 120 & 24-36 hours & 0.0 & 41 & {$[14]$} \\
\hline \multirow{2}{*}{ IND-Zn } & \multirow{2}{*}{$1(\mathrm{WCP})$} & \multirow{2}{*}{131} & 3 days & 1.0 & 63 & \multirow{2}{*}{ [15] } \\
\hline & & & 1 month & 0.0 & 67 & \\
\hline IND-Zn & \multirow{3}{*}{1 (Train Tickets) } & \multirow{3}{*}{$\mathrm{N} / \mathrm{A}$} & \multirow{3}{*}{ 9-10 months } & 10 & 29 & \multirow{3}{*}{ [16] } \\
\hline DFO & & & & 19 & 23 & \\
\hline Ninhydrin & & & & 24 & 27 & \\
\hline DFO & $\begin{array}{c}1 \text { WCP } \\
1 \text { craft paper }(\mathrm{CP}) \\
1 \text { cardboard (Ca) } \\
1 \text { recycled paper (RP) } \\
1 \text { thermal paper(TP)) }\end{array}$ & 8 & 1-28 days & $\begin{array}{c}\text { (WCP) } 0.36 \\
\text { (CP) } 94 \\
\text { (Ca) } 30 \\
\text { (RP) } 6.0 \\
\text { (TP) } 64\end{array}$ & $\begin{array}{l}\text { (WCP) } 45 \\
\text { (CP) } 0.71 \\
\text { (Ca) } 7.5 \\
\text { (RP) } 13 \\
\text { (TP) } 3.9\end{array}$ & {$[10]$} \\
\hline Physical Developer & \multirow{2}{*}{$1 \mathrm{WCP}$} & \multirow{2}{*}{148} & $\begin{array}{l}36 \text { hours } \\
30 \text { days }\end{array}$ & $\begin{array}{l}30 \\
29\end{array}$ & $\begin{array}{l}17 \\
14 \\
\end{array}$ & \multirow{2}{*}{ [17] } \\
\hline Oil Red O & & & $\begin{array}{l}36 \text { hours } \\
30 \text { days }\end{array}$ & $\begin{array}{l}63 \\
89\end{array}$ & $\begin{array}{l}2.0 \\
1.0\end{array}$ & \\
\hline $\begin{array}{l}\text { Vacuum metal } \\
\text { deposition }\end{array}$ & \multirow{2}{*}{$\begin{array}{c}4 \text { (Black bin bags, Orange } \\
\text { carrier bags, Clear sandwich } \\
\text { bags, Cowlings) }\end{array}$} & \multirow{2}{*}{7} & \multirow{2}{*}{ 2-28 days } & $15 \dagger$ & 57 & \multirow{2}{*}[18]{} \\
\hline $\begin{array}{c}\text { Small Particle } \\
\text { Reagent (Wetwop }{ }^{\mathrm{TM}} \text { ) }\end{array}$ & & & & $17 \dagger$ & 62 & \\
\hline $\begin{array}{c}\text { Cyanoacrylate } \\
\text { (CA) } \\
\text { Cyanoacrylate }+ \\
\text { Rhodamine 6G } \\
(\mathrm{CA}+\mathrm{R} 6 \mathrm{G})\end{array}$ & $\begin{array}{c}3 \text { (Glossy Cardboard, } \\
\text { Polystyrene, Ziplock Bags) }\end{array}$ & 3 & $1-8$ weeks & $\begin{array}{c}51(\mathrm{CA}) \dagger \\
28(\mathrm{CA}+\mathrm{R} 6 \mathrm{G}) \dagger\end{array}$ & N/A & [19] \\
\hline
\end{tabular}


106 When a mark is detected, it is important to understand the differences in fingermark quality 107 and the reporting of these differences. A fingermark can be detected if there is enough 108 material for the detection technique to interact with, either by chemical or physical means. In 109 fingermark detection research, the more commonly reported classification for detected marks 110 is whether a mark is identifiable or not (since this is of the most value to practitioners), but 111 the factors that make a fingermark identifiable are complex. The quality of a developed mark 112 is not just dependent on the detection technique, the donor, or fingermark age, but also other 113 factors such as the amount of deposition force, portion of the finger touching the substrate, 114 distortion, and potential substrate interferences including preferential transfer of some 115 secretions. There is another layer of complexity when the variability of the assessor is 116 considered. Two different fingermark examiners may have different opinions on the quality 117 of a fingermark [20]. Added to this is the inherent subjectivity with respect to the assessment 118 scales themselves and the assessment process. There is variability depending on whether the 119 assessment is done via direct visualisation of the developed marks (variable results depending 120 on the light source and filters, for example) or via an assessment of images. With the latter, it then depends on how the images are captured, processed, and presented to the assessor. If the assessment is not "blind" then there is the potential issue of contextual bias (i.e., a subconscious and unintentional "push" towards the expected or desired result). The complexity of all of the factors involved is reinforced by the data in Table 1 where there is significant variability in the number of identifiable marks reported in each study, even when the same technique is applied.

128 While several studies have looked at introducing more consistent methods for fingermark deposition to increase reliability of results and to minimise variability - largely focusing on controlling the deposition force and contact angle between the fingertip and the surface, which are the easier parameters to control [21-23] - there has been no consensus regarding

132 the best approach to address this issue. Reducing fingermark variability at an early stage of 133 the research may be an appropriate method to speed up the process; however, since 134 fingermarks are variable by definition, variability is a parameter that must be taken into consideration when validating techniques. Variability needs to be considered as being unavoidable in such studies, so it should be factored into the research design. 
138 In order to get a better understanding of detection techniques, to better evaluate new methods,

139 and to obtain more accurate estimates of detection rates, more research needs to be performed

140 examining the fundamentals of fingermark development. The aim of this study was to

141 establish a better understanding of the factors that affect fingermark detection. The following

142 factors; effect of substrate, donor (inter and intra variability), depletion and hand or finger

143 type were studied in this research. By gaining a better understanding of these effects,

144 recommendations can be made to improve experimental design in future fingermark research

145 studies.

\section{Materials and methods}

\section{General Methodology}

149 The study looked at the effect that substrate, donor, and depletion number have on the quality

150 of developed marks using two routine fingermark detection techniques, indanedione-zinc

151 (IND-Zn) reagent and cyanoacrylate fuming with rhodamine 6G staining (CA+R6G). The

152 most common routine detection methods in Australia (IND-Zn for porous and CA+R6G for

153 non-porous substrates) were used so that results could be related to operational casework. The

154 collection of latent fingermarks and the selection of test substrates were performed in 155 accordance with the guidelines published by the International Fingerprint Research Group 156 (IFRG) [11].

\section{Fingermark deposition}

159 Natural fingermarks from five donors (four males, one female) were collected at random 160 times of the day, during normal activities and without any finger preparation (i.e., no additional 161 washing of hands and no artificial "charging" of the fingers with secretions). No specific instructions were given to the donors as the intention was to collect realistic marks. Each donor deposited ten fingermarks (one from each finger) in a depletion series of four marks

164 (40 marks in total). This process was repeated a minimum of five times per substrate and

165 donor, but a maximum of four sets of fingermarks were collected per donor per day with

166 a minimum time period of one hour between each deposition. Fingermarks were kept in an

167 uncontrolled laboratory environment (Mean temperature $19.5+/-1^{\circ} \mathrm{C}$, mean relative 168 humidity $54.3+/-15 \%$ ) and stored in a drawer for either three days or seven days before development. 


\section{Substrates}

172 Fingermarks were deposited on two porous surfaces (Reflex 100\% Recycled A4 white paper

173 and Reflex Ultra white A4 premium paper) and two non-porous surfaces (Marbig A4

174 polypropylene sleeves and Hercules large polyethylene ziplock bags). A grid template was

175 printed on the paper sheets prior to fingermark collection. Both plastic surfaces were used as

176 received, with no pre-cleaning and a grid template was inserted into the sleeve or bag prior to

177 fingermark collection. A breakdown of the number of latent marks deposited is provided in

178 Table 2. While the number of fingermark replicates collected for each donor was not consistent

179 (due to donor availability), there were sufficient marks for each substrate and fingermark

180 age for each donor in order to make valid comparisons, with a minimum of 200 fingermarks

181 deposited for each set of conditions.

\begin{tabular}{|c|c|c|c|c|c|c|c|}
\hline AGE & SUBSTRATE & Donor A & Donor B & Donor C & Donor D & Donor E & Total \\
\hline \multirow{3}{*}{ 3-day } & PREMIUM & 400 & 280 & 200 & 400 & 400 & $\mathbf{1 6 8 0}$ \\
\cline { 2 - 8 } & RECYCLED & 480 & 440 & 400 & 440 & 200 & 1960 \\
\cline { 2 - 8 } & ZIPLOCK & 360 & 280 & 200 & 400 & 360 & 1600 \\
\cline { 2 - 8 } & PL-SLEEVE & 480 & 440 & 440 & 400 & 200 & 1960 \\
\hline 7-day & PREMIUM & 400 & 240 & 360 & 320 & 400 & $\mathbf{1 7 2 0}$ \\
\cline { 2 - 8 } & RECYCLED & 480 & 200 & 480 & 400 & 320 & $\mathbf{1 8 8 0}$ \\
\cline { 2 - 8 } & ZIPLOCK & 400 & 200 & 360 & 320 & 400 & $\mathbf{1 6 8 0}$ \\
\cline { 2 - 8 } & PL-SLEEVE & 480 & 200 & 440 & 320 & 360 & $\mathbf{1 8 0 0}$ \\
\hline
\end{tabular}


agent and rhodamine 6G (R6G) staining solution were prepared and applied as outlined Fin by Champod et al. [24]. Fingermarks deposited on the porous substrates were developed

ger

ma

rk

dev

elo

pm

ent

$\mathrm{T}$

h

e

i

n

d

a

n

e

d

i

o

n

e

i

n

c

$\mathrm{N}$

D .

.

d
with IND-Zn. Fingermarks deposited on the non-porous substrates were developed with cyanoacrylate (CA) and subsequently stained with R6G. IND-Zn development was achieved by placing the treated items in an ironing press at $160^{\circ} \mathrm{C}$ for $10 \mathrm{~s}$. CA fuming was performed using a Foster+Freeman MVC 1000D fuming cabinet, using $0.5 \mathrm{~g}$ of cyanoacrylate $\left(\right.$ Cyanobloom ${ }^{\mathrm{TM}}$; Foster+Freeman, UK) per fuming cycle with heating at $120^{\circ} \mathrm{C}$, a fuming time of $20 \mathrm{~min}$, and $80 \%$ relative humidity in the cabinet.

Cyanoacrylate developed fingermarks were cured for 24 hours before being stained with 
R6G, rinsed with water, and allowed to dry. IND-Zn and CA+R6G developed fingermarks were visualised and photographed in the luminescence mode using a Poliview ${ }^{\circledR}$ IV forensic imaging system (Rofin Australia Pty. Ltd., Australia), with a Polilight PL550XL forensic light source operating at $530 \mathrm{~nm}$ and with a $610 \mathrm{~nm}$ band-pass barrier filter on the camera for the R6G and with a $590 \mathrm{~nm}$ band-pass barrier filter on the camera for the IND-Zn.

\section{Fingermark evaluation and analysis}

202 Developed fingermarks were assessed using the Home Office CAST scale [25] (Table 3).

203 Three independent assessors (with >5 years of fingermark detection research experience), not 204 involved in the detection and recording of the fingermarks used in the study, graded each mark in a random order. All scores from the assessors were collated and the median score for each fingermark was calculated. Each fingermark score was then classified according to donor (both inter and intra variability), substrate, age, finger and depletion number. Due to the large number of specimens and in the interests of consistency between assessors, it was decided before the evaluation phase that a mark that was considered identifiable was given a score of 3 or 4, while fingermarks that had some ridge detail but not enough to be considered identifiable were granted a score of 2 . Based on this, the median fingermark scores were grouped into the categories specified in Table 4, and from this trends related to the examined

213 factors could be determined.

\begin{tabular}{|c|c|}
\hline Score & Qualitative equivalent \\
\hline 0 & No evidence of mark \\
\hline 1 & $\begin{array}{c}\text { Weak development; evidence of contact, but no ridge } \\
\text { detail }\end{array}$ \\
\hline 2 & $\begin{array}{c}\text { Limited development, about } 1 / 3 \text { of ridge details are } \\
\text { present, but probably cannot be used for identification } \\
\text { purposes }\end{array}$ \\
\hline 3 & $\begin{array}{c}\text { Strong development; between } 1 / 3 \text { and } 2 / 3 \text { of ridge details; } \\
\text { identifiable fingermark }\end{array}$ \\
\hline 4 & $\begin{array}{l}\text { Very strong development; full ridge details; identifiable } \\
\text { fingermark }\end{array}$ \\
\hline
\end{tabular}




\begin{tabular}{|c|c|}
\hline Grouped Score & Qualitative equivalent \\
\hline 0 & No evidence of mark \\
\hline $1-2$ & Detected, but not suitable for comparison \\
\hline $3-4$ & Suitable for comparison \\
\hline
\end{tabular}

\section{$220 \quad$ Results and discussion}

\section{General results}

222 Overall, 14,280 fingermark images were graded and sorted into the three groups presented in 223 Table 4. When the data from all donors, techniques and substrates are analysed together, only $22411.7 \%$ of marks were not detected (0 score) and $28.9 \%$ were suitable for comparison (score 225 of 3-4). The remaining 59.4\% were detected but with an insufficient quality to be used in the comparison process (score of 1-2). While this particular study may be considered 'preliminary' in terms of the number of fingermark age, donor and substrate variables, the inclusion of 14,280 processed and assessed impressions (ranging from strong to weak) provides a robust model population. This scale of fingermark collection is rarely met outside of long-term operational or pseudo-operational validation research, where the focus is on blind trials using discarded items or genuine exhibits; replicate marks from the same donor (if any are present) are often depletions and the true number of depositions is unknown. The large known population of replicates presented in this study gives some insight into the degree of variation in fingermark quality that may be expected from the same individuals over several weeks following a semi-controlled process. .

237 The results from this study reaffirm the complexity of fingermark residues and their detection. Some of the results agree with our understanding of fingermark detection, while other results provide interesting observations warranting further research. The results presented below are a collation of both fingermark ageing periods (three days and seven days) as there was no significant difference in the number of marks recovered for each period (Figure 1). This result is not surprising given the small time difference between the two age points. While there is a slight increase in the number of zero scores (no development) and a decrease in the number of 3-4 (identifiable marks) at seven days, the effect is minor. Future studies of this nature should expand the age differences between samples to gain a better 
understanding of the relationship between the substrate type, the age of the fingermark, and the success of fingermark detection methods.

Figure 1: Collated results for all donors and substrates developed 3 and 7 days after deposition and an overall total

\section{Effect of substrate}

The substrate that a fingermark is deposited on will determine the choice of detection technique and there is a general assumption that IND-Zn and CA+R6G are the techniques most likely to recover high quality marks, as they are routinely used as start-of-sequence techniques following optical examination. As seen in Figure 2, there was a higher proportion of marks not detected on the non-porous substrates than on the porous substrates. Similarly, the developed marks on porous substrates tended to be of a higher quality than the fingermarks developed on the non-porous surfaces. This result could be due to several factors, including intrinsic surface differences where fingermarks deposited on paper will be quickly absorbed into the matrix and protected, whereas on non-porous substrates they are more likely to be damaged from the environment and friction when handled. This could also be due to a more sensitive reaction between IND-Zn and the latent fingermark components compared to the CA fuming process. 


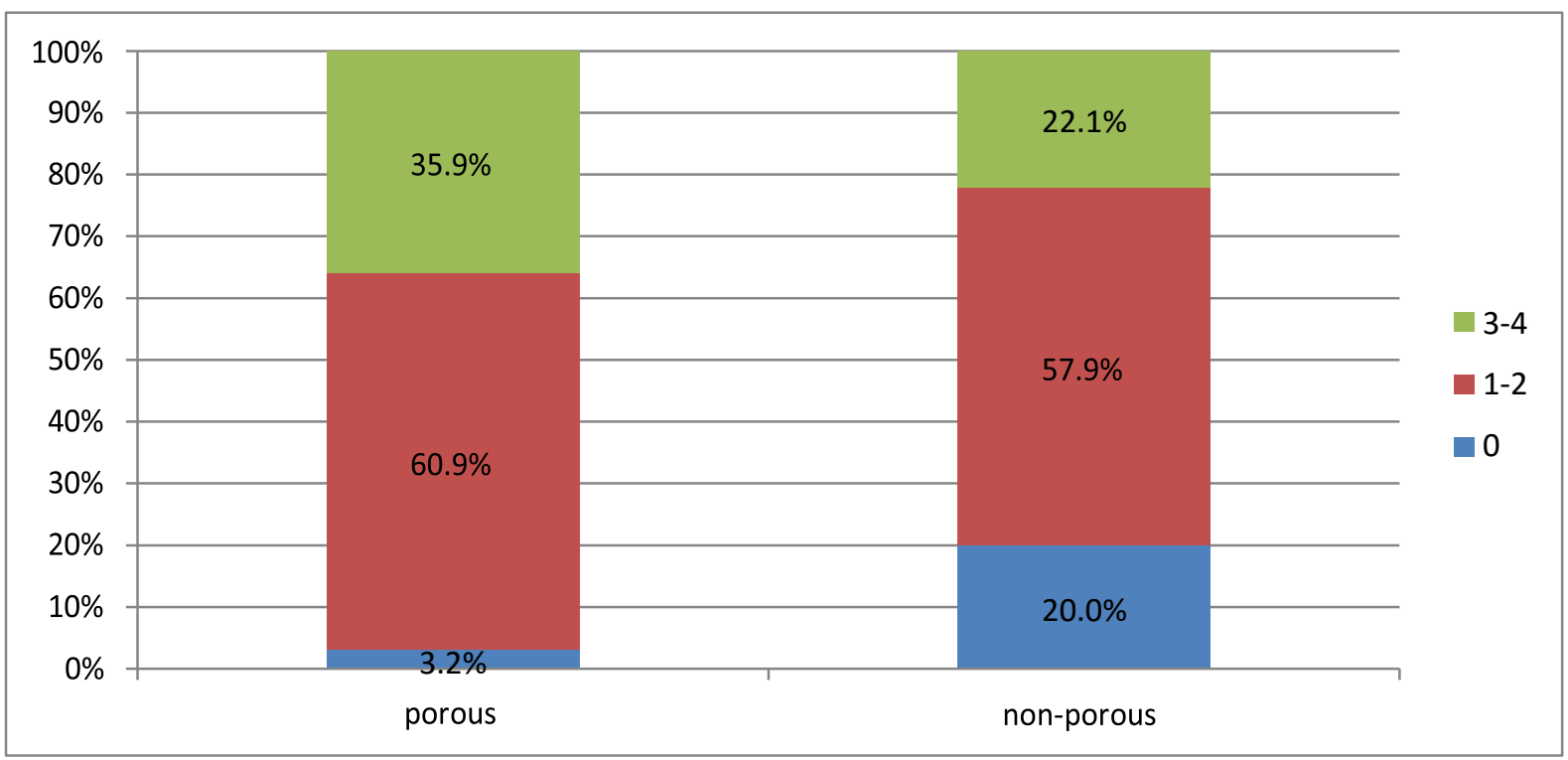

Figure 2: Comparison of the number of developed marks for all donors on porous and non-porous surfaces $(0=$ no evidence of mark; 1-2 = detected but not suitable for comparison; 3-4 = suitable for comparison).

275 When the substrates are separated out (Figure 3), there is very little difference between the 276 recycled and premium brands of paper. However, a clear difference can be seen with the non277 porous results, with more fingermarks and higher quality marks being developed on the 278 ziplock bags than on the plastic folder sleeves. This result reinforces previous studies which 279 demonstrated that different types of plastic products behave differently with respect to 280 fingermark detection methods, even when the materials share the same base polymer [26, 27].

281 The difference shown here is quite obvious, with $10 \%$ more marks going undetected on the 282 plastic sleeves, which would justify further research into non-porous substrate interactions 283 particularly plastics - with fingermark detection techniques. Previously, there has been a 284 correlation observed in other studies between paper type and fingermark quality [10, 28, 29].

285 This was not observed in this study; however, the two paper types were limited to the same 286 brand and manufacturer, meaning that they may not be representative of other papers available on the market. More plastic and paper types need to be included in subsequent studies in order to better encompass substrate variability. 




Figure 3: Comparison of developed marks for all donors on each surface $(0=$ no evidence of mark; $1-2=$ detected but not suitable for comparison; 3-4 = suitable for comparison).

\section{Effect of Donor (inter- and intra-donor variability)}

The variation in fingermark composition between individuals is common knowledge amongst fingermark researchers $[30,31]$. This is one of the main reasons for having multiple donors depositing fingermarks when assessing the viability of a new technique [11]. Generally, donors are classified into three broad groups - strong, medium and weak - with strong donors more likely to produce higher quality marks, while weak donors are more likely to produce poorer quality marks. In most fingermark detection research, these categories are assigned to a donor either by the researcher, based on prior results, or self-assessed by the donor. The results in Figure 4 and Figure 5 demonstrate that the donor variability is a lot more nuanced than the three categories allow for. An example of this can be seen when the results for donors $\mathrm{A}$ and $\mathrm{E}$ are compared; they have very similar percentages of undetected marks but there is a significant difference ( $51 \%$ vs $8 \%$, respectively) between the two donors in terms of the number of identifiable marks.

This raises the question of whether a donor is classed as a weak donor because they have a high number of undetectable marks (zero scores) or because they have a low number of potentially identifiable impressions (3-4 scores). With only $8 \%$ of donor E's processed fingermarks scoring 3-4 on the CAST scale, the assignment of weak donor status based on low number of potentially identifiable marks appears relatively straightforward. For "moderate" donors, this question becomes more difficult to answer. Transfer factors such as 
314 smudging or excessive deposition force can also influence the number of traces assessed as

315 being potentially identifiable. In these instances, such as for donor D, the donor may be a

316 strong secretor but a poor transferrer, being a failure at deposition rather than a failure at 317 detection.

318

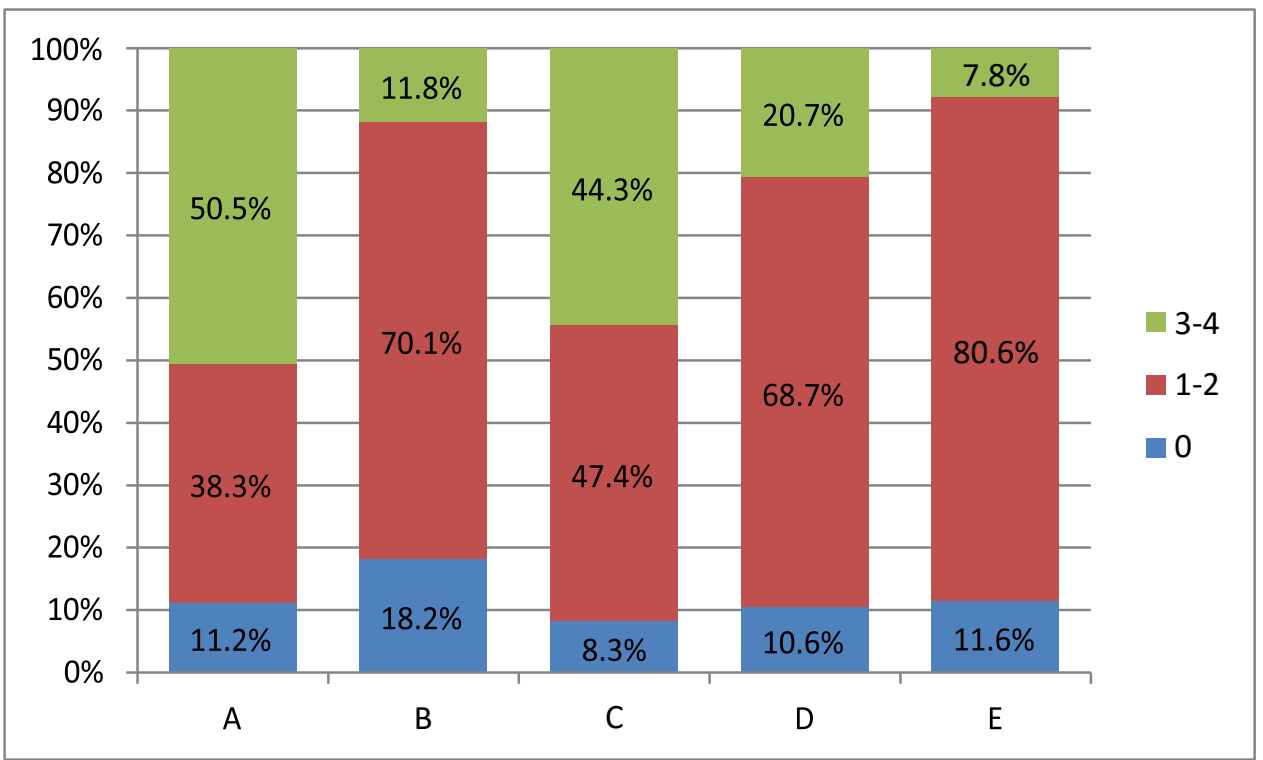

Figure 4: Comparison of the overall number of developed marks on all surfaces for each donor $(0=$ no evidence of mark; 1-2 = detected but not suitable for comparison; 3-4 = suitable for comparison).

When each donor's marks are separated out by surface type (and, hence, detection technique), we see a more pronounced effect on the quality of marks recovered (Figure 5). Fingermarks from some donors appear to work better for certain techniques; e.g., donor A has the higher number of identifiable marks on porous substrates, but the $2^{\text {nd }}$ highest number of marks not detected on non-porous substrates. These results indicate that the deposition 'strength' for certain donors is technique-dependent; for example, a donor's secretions may contain more amino acids, resulting in higher-quality IND-Zn marks, but less of the components targeted by CA. 


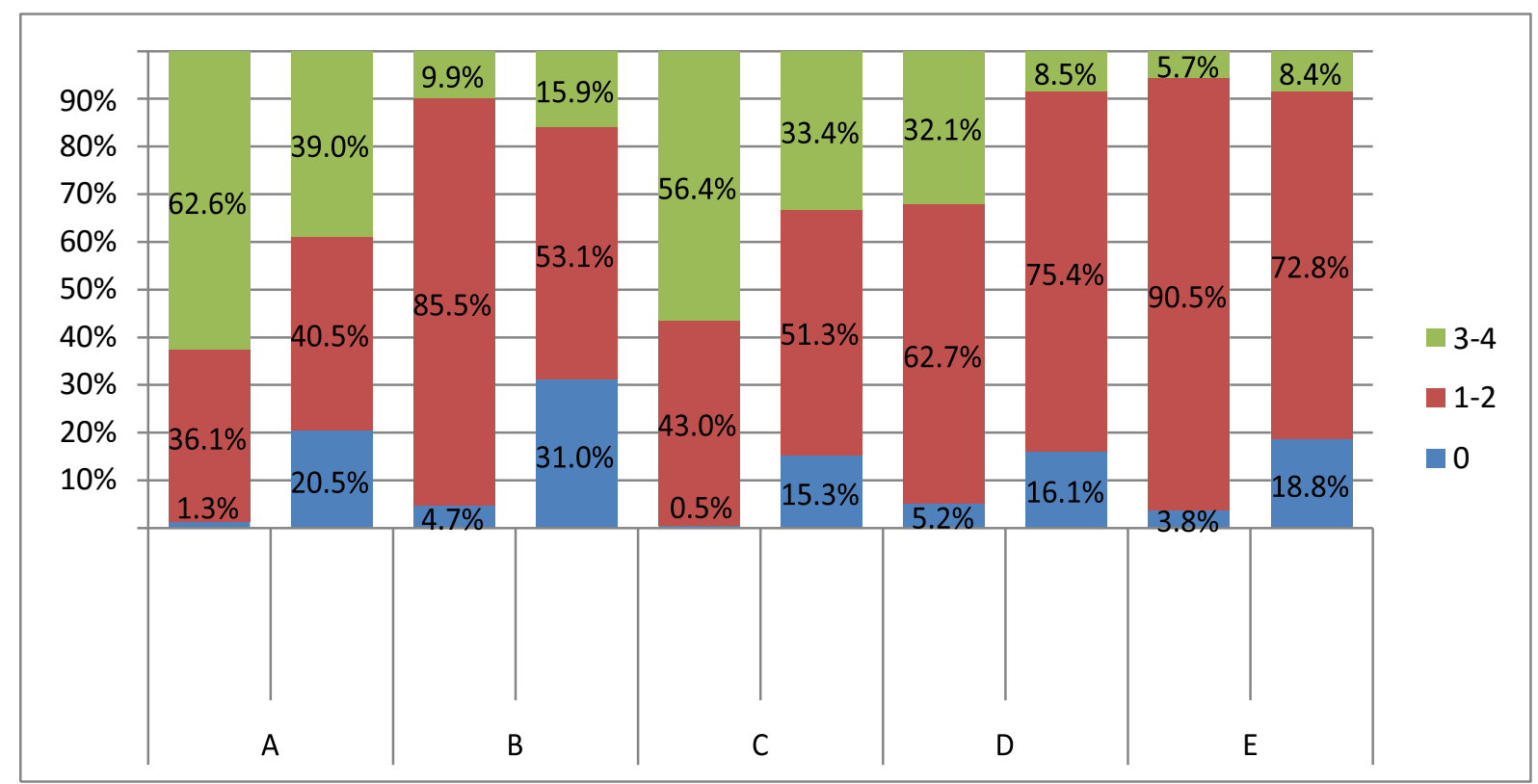

332

333

334

335

336

337

338

339

340

341

342

343

344

345

346

347

348

349

Figure 5: Comparison of the number of developed mark for each donor and for each substrate type $(0=$ no evidence of mark; 1-2 = detected but not suitable for comparison; 3-4 = suitable for comparison).

To examine the repeatability of a donor producing similar quality marks across different repeats (only the first deposition of each set was taken into consideration to avoid any variation deliberately induced by a depletion series), data was compared and analysed for all surfaces. The results for Reflex Ultra white A4 premium paper and Marbig A4 plastic sleeves are presented in Figure 6 and Figure 7, respectively. Results within each substrate type showed similar variabilities for donors but there is a pronounced difference when comparing between substrate types. In regard to the porous substrates, the donors had a tendency to be consistent with the number of marks not detected; however, donors (particularly in the case of donor C) were variable with the number of detected and identifiable marks. This contrasts with the non-porous surfaces, which showed that there was variability of most donors across all grading classifications, with some donors more consistently producing identifiable fingermarks. These results demonstrate that, even when the same donor deposits marks on the same type of surface, the quality and number of developed marks is extremely variable. This reinforces the need for a large number of marks to be analysed for any fingermark study, whether this be in the form of replicates or large donor pools. 


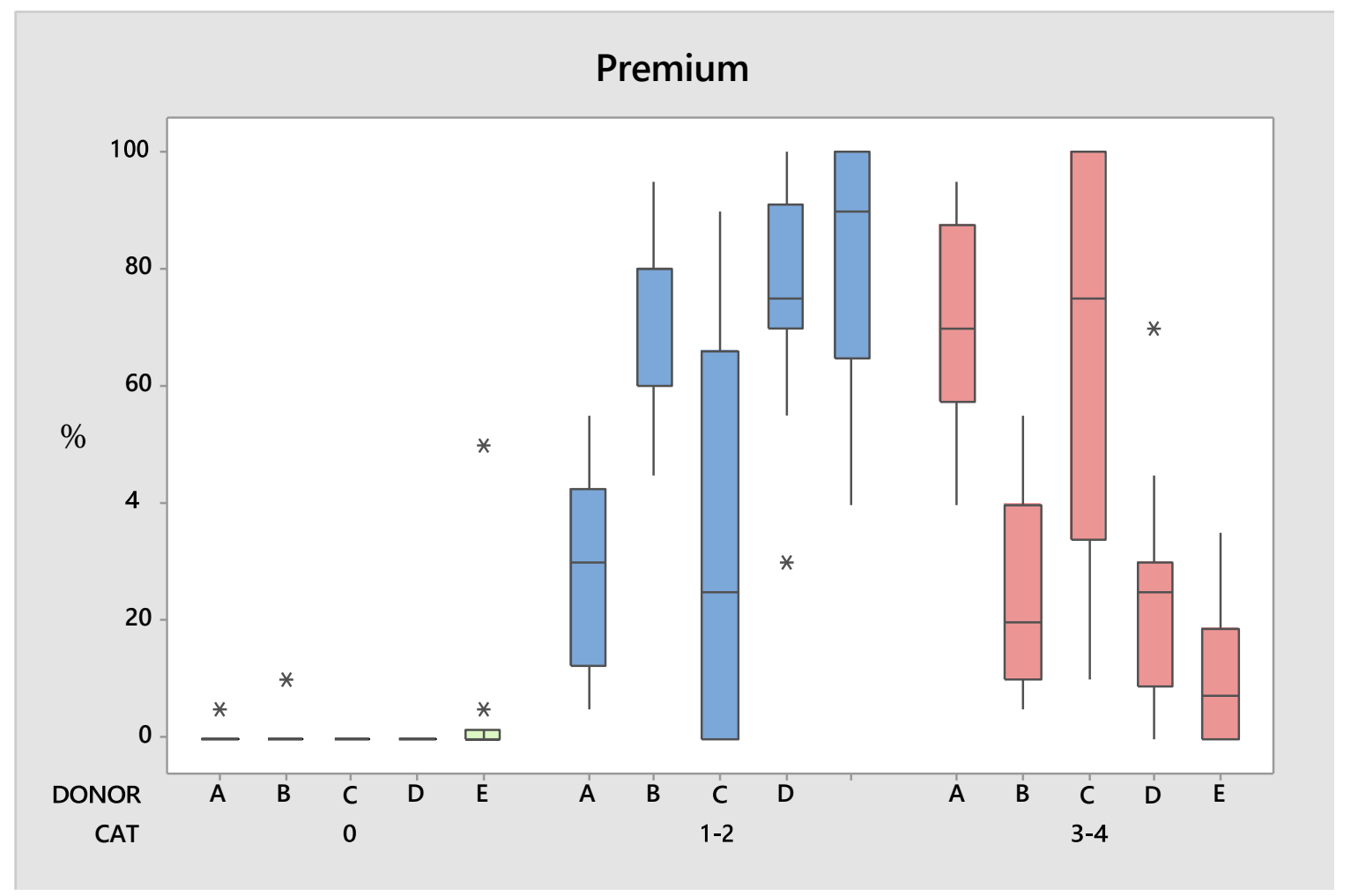

Figure 6: Summary of intra-donor variability for repeated depositions for each donor on premium white paper $(0=$ no evidence of mark; 1-2 = detected but not suitable for comparison; 3-4 = suitable for comparison).

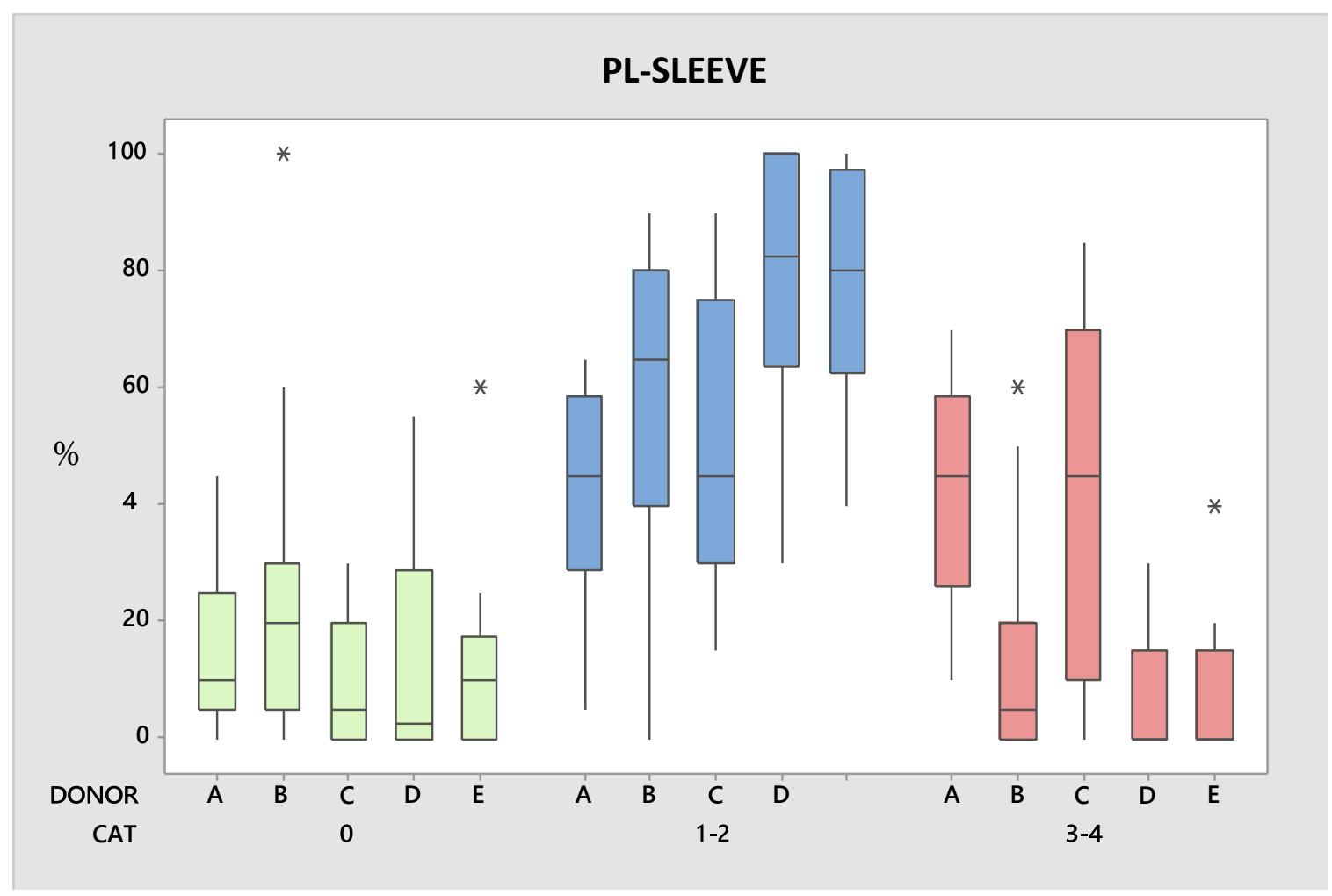


Figure 7: Summary of intra-donor variability for repeated depositions for each donor on plastic folders $(0=$ no evidence of mark; 1-2 = detected but not suitable for comparison; 3-4 = suitable for comparison).

\section{Effect of Depletion}

359 Depletion series are commonly used in fingermark research to demonstrate the effectiveness 360 of a technique to develop marks with lower concentrations of residues. It is commonly 361 hypothesised that the quality of deposited fingermarks will degrade with subsequent depletions, provided that there is no opportunity for the fingermark secretions to reequilibrate or replenish between depositions. Figure 8 shows that, as the number of depletions increases, there is a decrease in the number of identifiable marks, and an increase in the number of no development scores. It is also interesting to note that, across the first 3 depositions, the percentage of detected marks not suitable for comparison does not change, with only a slight increase at the $4^{\text {th }}$ deposition. The results suggest that sequential depositions are a good way to evaluate a detection technique's relative sensitivity as increasingly weaker marks are produced.

To further investigate this relationship, all first deposition marks that were graded with a 0 , 1-2 or 3-4 were respectively extracted, then the subsequent depletions in each series were analysed to determine if the initial scores remained consistent or they changed as the depletion number increased. These results are displayed in Figure 9 to 11. The impact of depletion number on fingermark quality appears to be a very complex situation, with a number of factors playing a role. An observable trend can be seen on non-porous (and to a lesser extent porous) substrates (when scored a 1-2 or 3-4 on the first depletion), with an increase in the number of undetected marks as the depletion increases. A small portion of marks originally graded 1-2 actually improved as the depletion increased (Figure 10); however, care should be taken when interpreting these results as there may have been other external factors such as deposition force and smudging of the initial mark that may have decreased the quality of the first impression. Interestingly, a similar observation was made for the marks originally graded a 0 , with some of the subsequent impressions in the series being of higher quality (Figure 11). This result is interesting as it implies that mark quality can improve with depletion; however, as with the previous case, further investigation into the specific marks should be conducted to understand the observed increase in quality. 


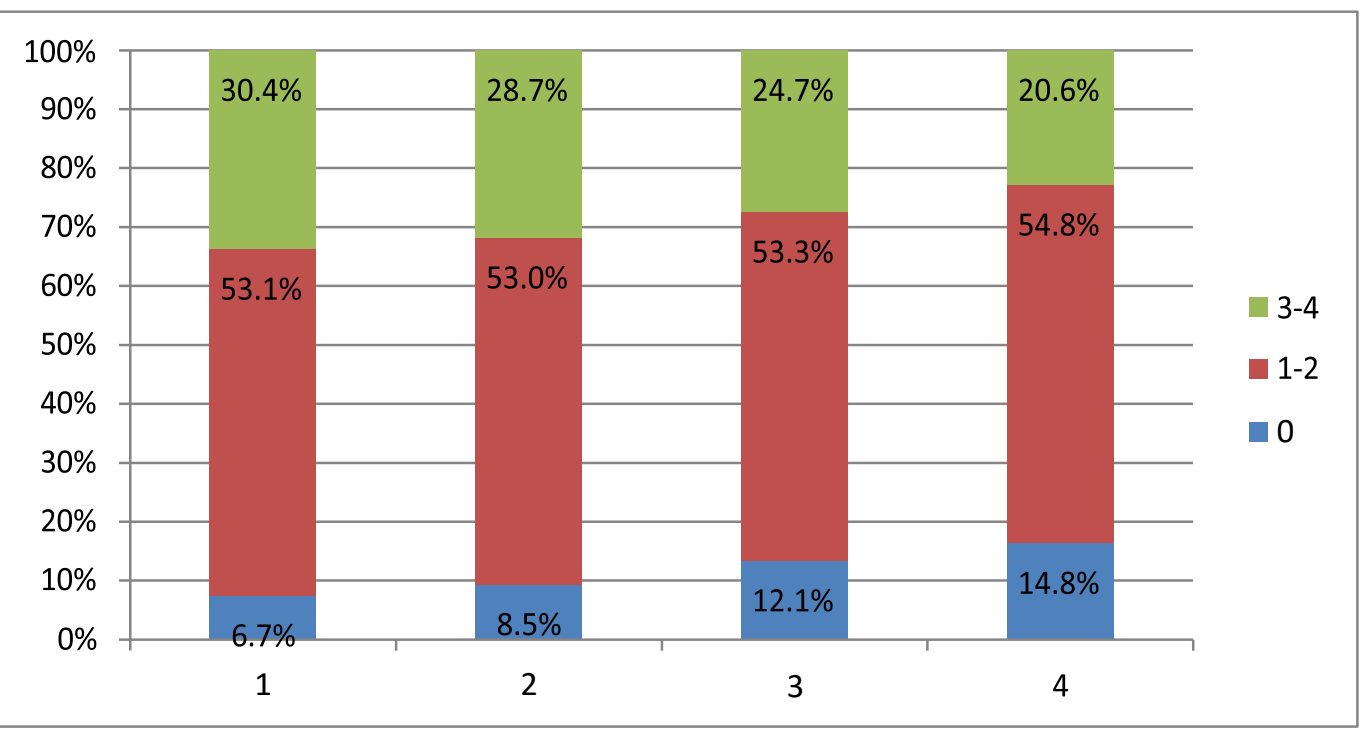

Figure 8: Collated scores for all fingermarks based on the depletion number (1 - 1st deposition, 2 - 2nd deposition, 3 390 3rd deposition, 4 - 4th deposition $)(0=$ no evidence of mark; $1-2=$ detected but not suitable for comparison; $3-4=$ 391 suitable for comparison).

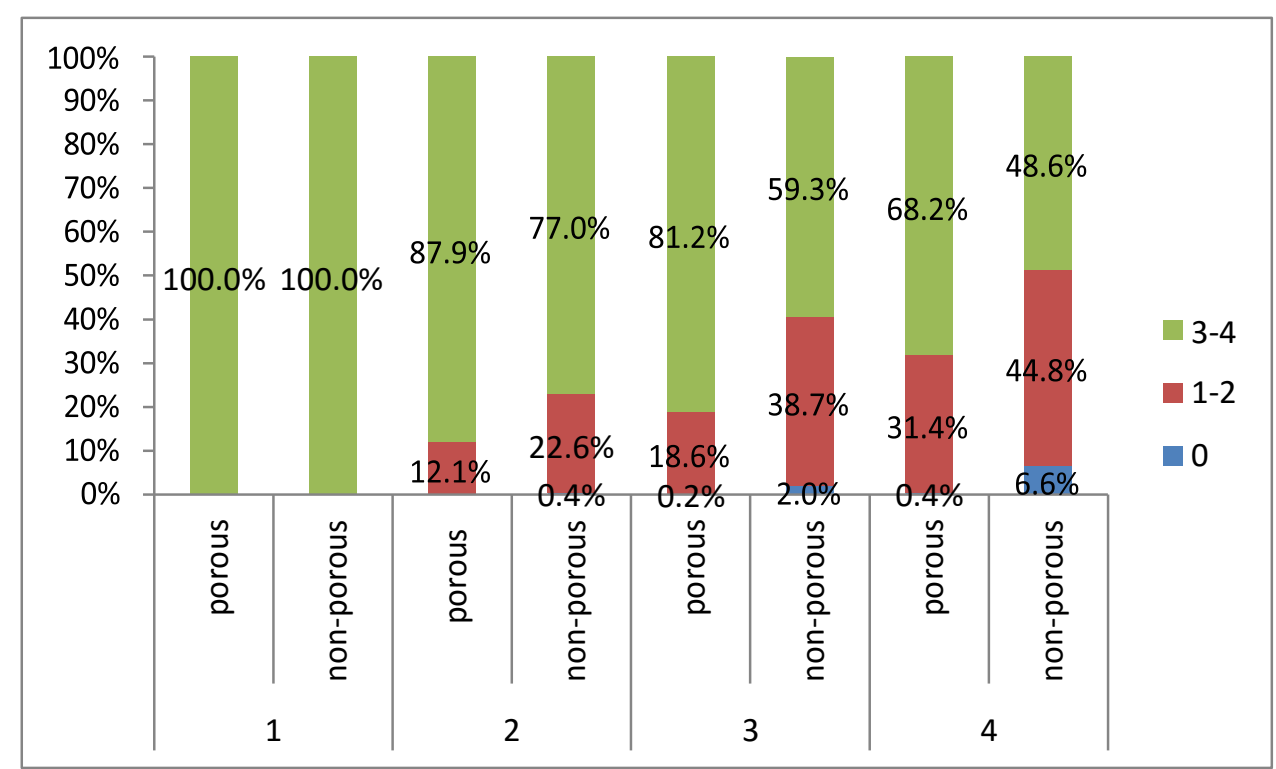

394 Figure 9: Collated scores for subsequent depletions for samples with an initial score of 3-4 for the first depletion (1 1st deposition, 2 - 2nd deposition, 3 - 3rd deposition, 4 - 4th deposition $)(0=$ no evidence of mark; $1-2=$ detected but not suitable for comparison; 3-4 = suitable for comparison). 


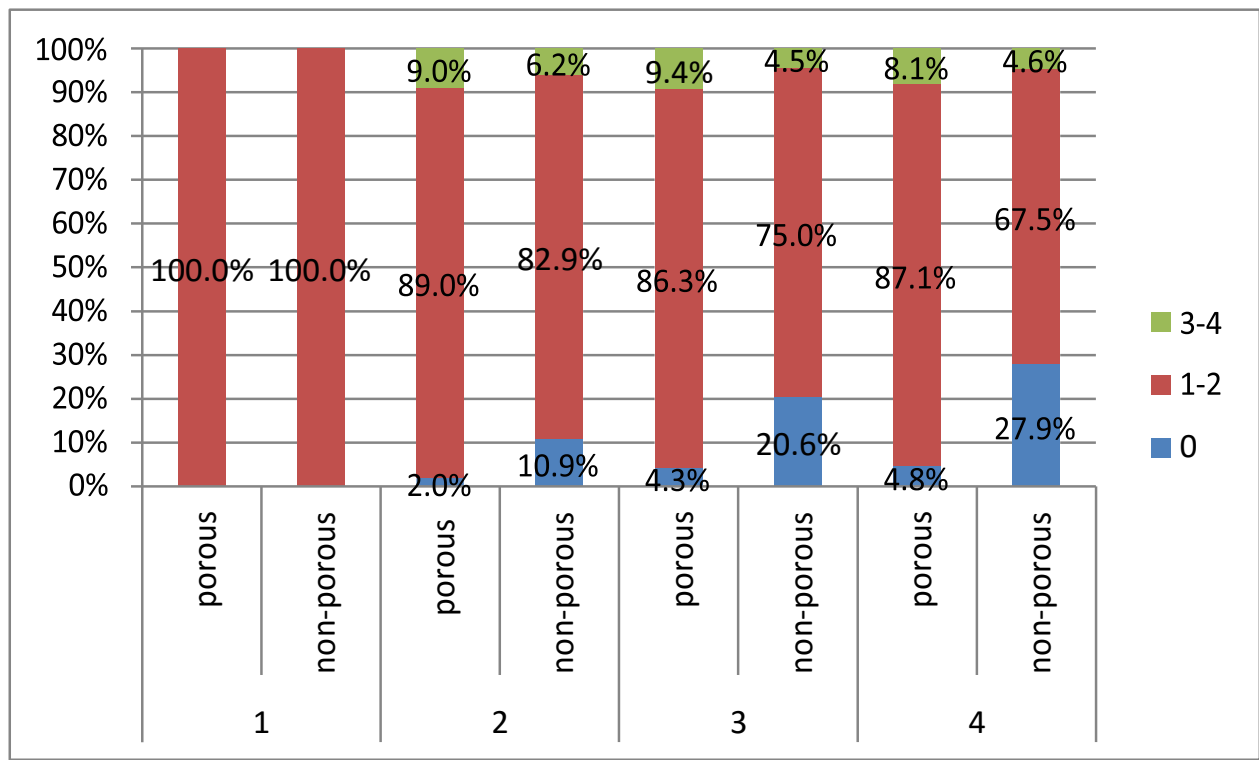

Figure 10: Collated scores for subsequent depletions for samples with an initial score of 1-2 for the first depletion 400

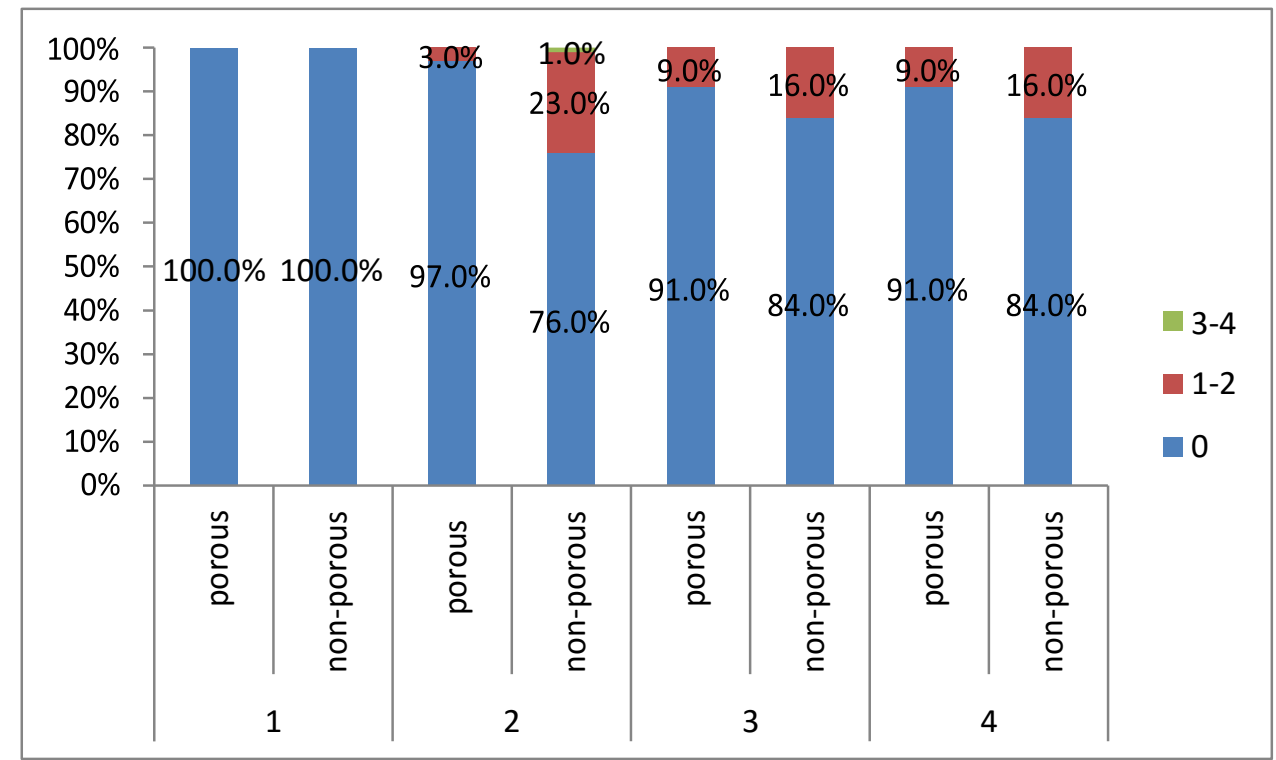

Figure 11: Collated scores for subsequent depletions for samples with an initial score of 0 for the first depletion

404 The number of marks not detected increased more rapidly on the non-porous surfaces than on 405 the porous substrates. This could indicate that the targets of the CA fuming do not replenish 406 fast enough between depletions. Whereas, the amino acids targeted by IND-Zn are either in a 407 high enough concentration to be detected over a wider range of depletions or they are quickly 408 replenished between depletions (at least to some extent). But, overall, the hypothesised trend 409 of increasingly weaker impressions in the depletion series is confirmed. 


\section{Hand and Finger type}

412 In order to determine whether there was a difference between impressions made with the left

413 or right hand, all scores were combined for each hand and compared (Figure 12). From this,

414 there are minor differences in the number of identifiable marks and marks detected but not 415 suitable for comparison (2 and $2.5 \%$ respectively).

416

417

418

419

420

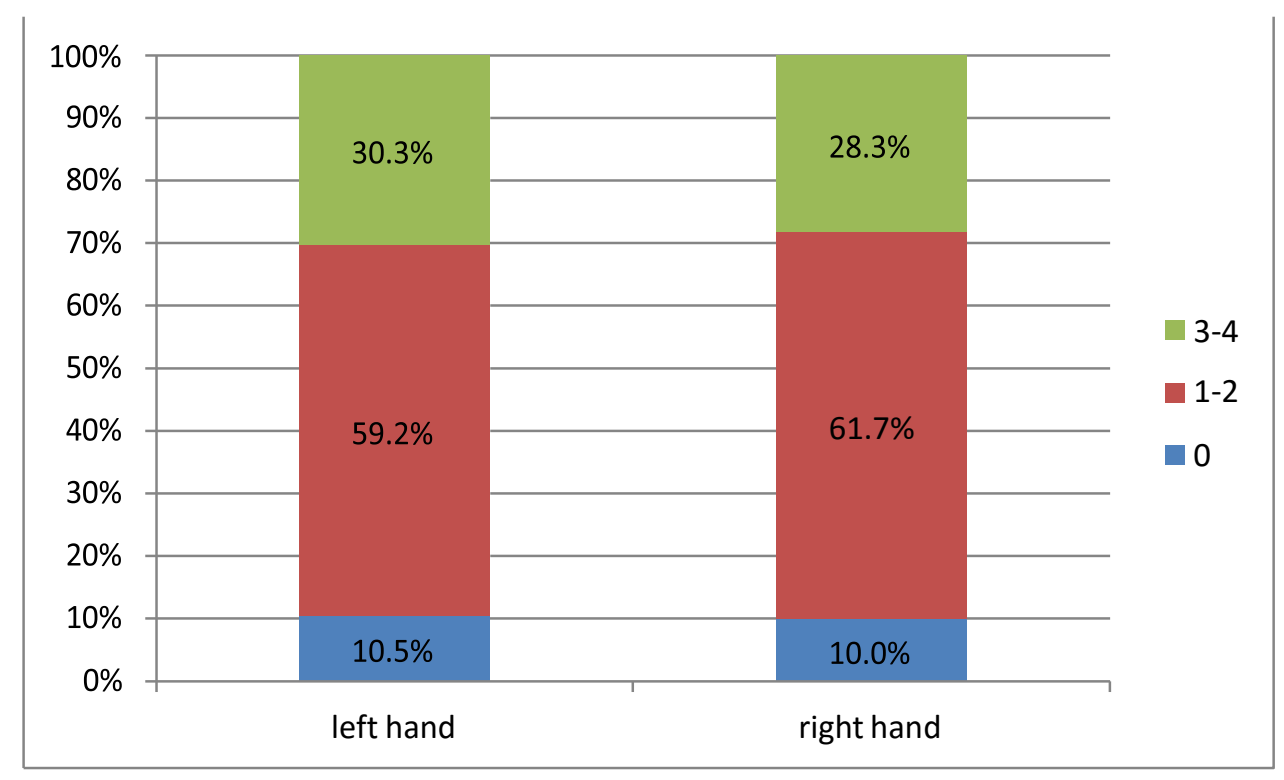

Figure 12: Comparison between the left and right hands. Collated scores for all donors and surfaces $(0=$ no evidence of mark; 1-2 = detected but not suitable for comparison; 3-4 = suitable for comparison)

421 When these values are separated out into the individual fingers, some interesting variations 422 between fingers and hands can be observed. Both hands have the highest number of 423 identifiable marks from the thumbs (compared to the other fingers from the same hand), 424 which, given their larger surface area, makes sense. Similarly, the little finger on each hand 425 has the lowest number of identifiable marks and the highest number of marks not detected. 426 When comparing between the hands, there is a larger variability in the number of identifiable 427 and detectable marks for the left hand compared to the right. It should be noted that all the 428 donors in this study were right handed so, while a comment cannot be made on the effect of 429 handedness on fingermark deposition, this may be an interesting avenue to explore in future 430 research. 


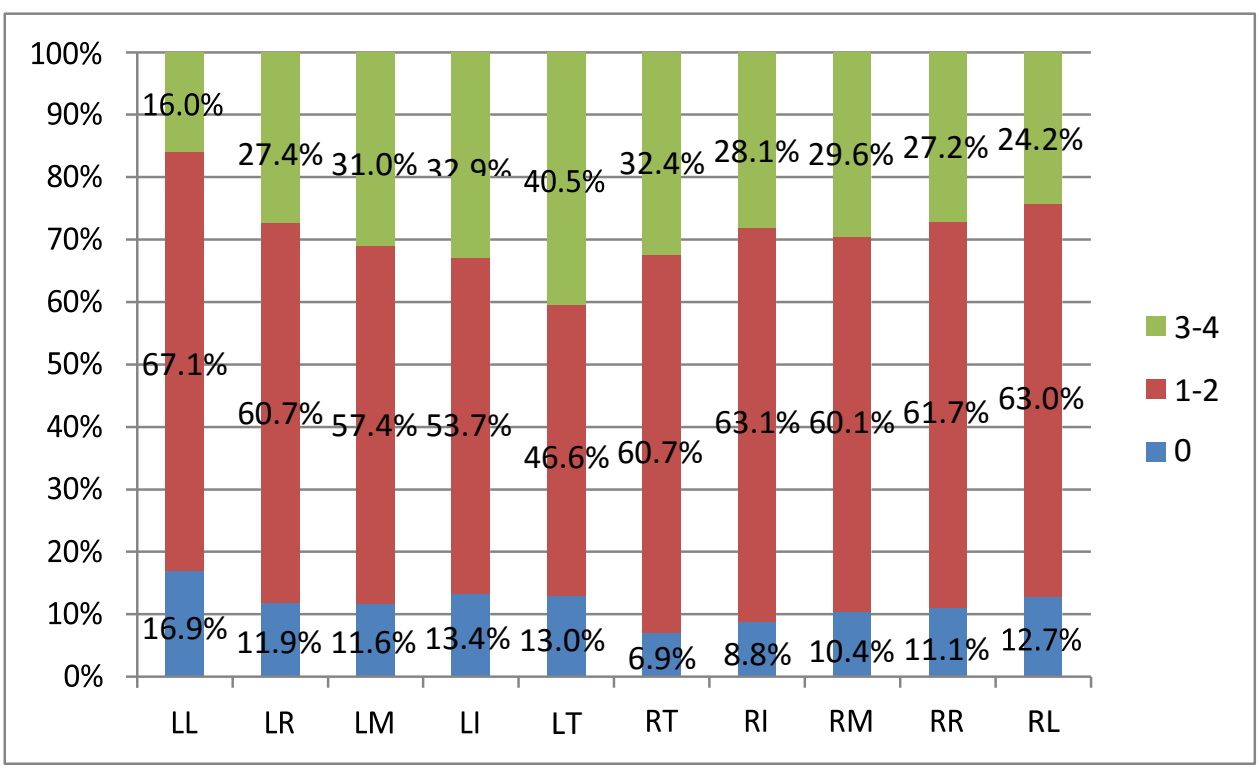

Figure 13: Comparison between the collated scores $(0$ - no evidence of mark; 1-2 detected, not suitable for comparison; 3-4 suitable for comparison) for all donors and surfaces by finger type $(L L=$ left little finger, $L R=$ left ring, $\mathrm{LM}=$ left middle, $\mathrm{LI}=$ left index, $\mathrm{LT}=$ left thumb, $\mathrm{RT}=$ right thumb, $\mathrm{RI}=$ right index, $\mathrm{RM}=$ right middle, $\mathrm{RR}$ $=$ right ring, $R L=$ right little finger)

\section{Discussion}

Gaining an understanding of the effectiveness of fingermark detection techniques continues to be of importance in forensic science research. Moreover, as there is increased scrutiny of the processes and reporting on the analysis of traces (in particular fingermarks), a better understanding of the effectiveness of methods and the limitations of those methods needs to be explored. This research has revealed some interesting findings, which challenge some of the previously held ideas in fingermark detection research. In particular, this work has indicated that the effectiveness of certain fingermark detection techniques is well above the $50 \%$ previous stated [8]. This work should be viewed as a step forward in our understanding; however, there is more work to be done in this area.

One of these areas is in our understanding of donor variability; the results (Figures 4 to 7) suggest that donor variability is more complex than initially believed. In particular, under controlled conditions and using the same donors over a short fingermark ageing period, there is significant variation in the quality of marks recovered. This raises some questions about how to best measure the effectiveness of fingermark techniques. The approach taken in this research was to use a small donor pool with a large number of repetitions. This approach, rather than employing a large donor pool with few repetitions, allowed for the observation of 
trends in relation to general factors such as the effect of depletion, donor intra-variability, and surface or donor dependency. However, this may not be suitable when investigating a new technique or method as there is value in investigating the effectiveness of a fingermark detection method on a larger population that would be more representative of operational casework.

\section{Evaluation of fingermark quality}

463 When undertaking a large-scale donor study, care must be taken when choosing an appropriate evaluation scheme for determining the quality of fingermark detection. The CAST scale is one of the most commonly used scales in this field to assist in qualifying the capability of a particular detection method; however, the limited ability of the scale to discern between a smudged fingermark with a strong reaction to a technique and an undistorted fingermark that is poorly detected should be taken into consideration when reporting findings. A fingermark can be assigned a value of one or two if there is evidence of a mark but insufficient detail for a comparison (no ridge detail for a score of one). The reason a mark can be given a score of one or two is not always dependent on the technique's ability to develop the mark. There were a number of cases in this study where the donor deposited marks with a higher amount of force or excessive secretions, which resulted in a loss of ridge detail and the developed mark being given a lower score (Figure 14). When compared to other marks assigned the same score, it can be seen that there is a clear discrepancy between cases where there are issues with the technique and cases where there are issues with the deposition. This can be potentially misleading when presenting results that are only based on quantitative scoring without taking into account the degree of development or enhancement produced by a technique, as this may understate the detection method's true ability to develop high quality fingermarks. It should be noted that similar shortcomings would be observed for other scoring methods in common use. A recommendation could be made to amend the current evaluation methods to have a qualifier for poorly developed (unidentifiable) marks categorised into poor development due to deposition issues or poor development due to poor fingermark enhancement by the technique to provide clearer reporting when assessing the ability of a technique to develop fingermarks. These results highlight the complexity associated with reporting fingermark research results and how misinterpretations are possible with respect to the effectiveness of a specific technique. 
Discrepancies between assessors' scores were minimised in this study by choosing three assessors and using the median of their scores. The evaluation process was performed independently and the three assessors were not involved in the fingermark detection and recording steps of the study. The marks were also assessed in a random order that was different for each examiner.
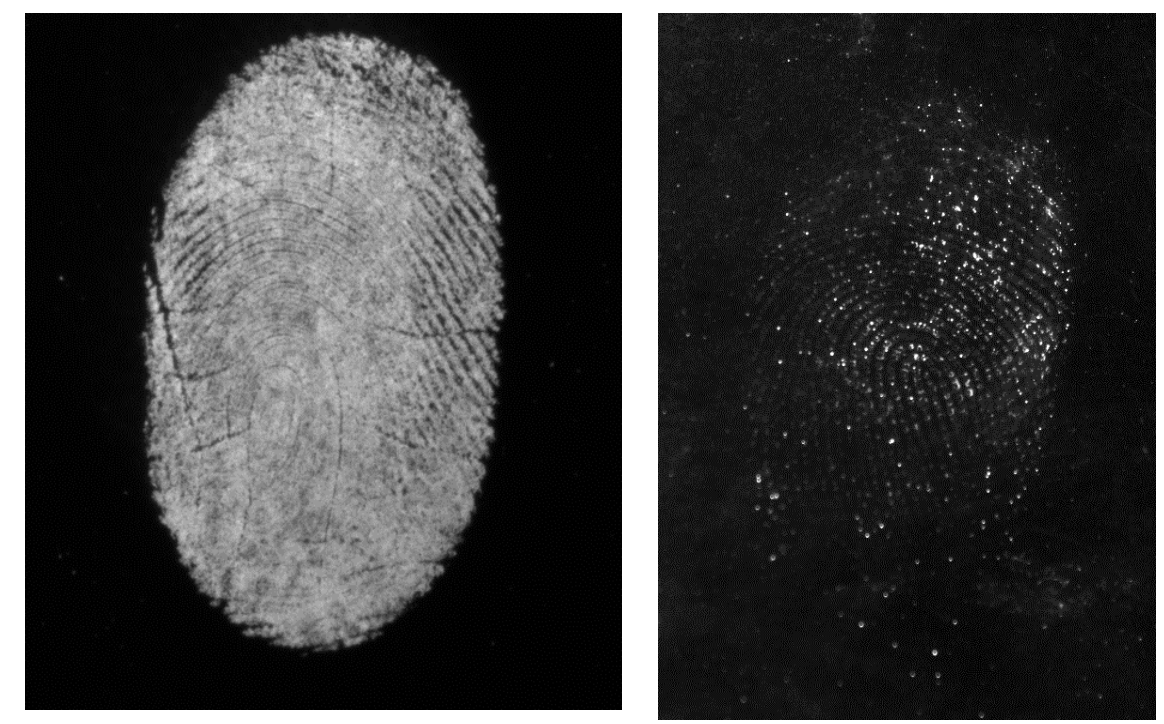

Figure 14: Representative images for fingermarks graded a score of two

\section{Impact of study}

508 The results of this study indicate that the currently employed methods IND-Zn and CA+R6G are able to detect a high proportion of fingermarks; the number of marks undetected on porous substrates is very low (3\%), while $20 \%$ of marks on non-porous substrates go undetected. These results are much lower than the reported 50\%; however, this needs to be interpreted in the context of the relatively fresh fingermarks processed in this study. Despite these promising figures, only $36 \%$ and $22 \%$ of fingermarks were deemed to be of identifiable quality for porous and non-porous surfaces, respectively. It would be expected that the actual success rates under casework conditions would be lower; the collection of fingermarks in this study was performed under semi-controlled conditions as the location of each fingermark was known and the samples were stored under laboratory conditions. The results reaffirm the need for ongoing fingermark detection research in order to increase success rates with respect to the recovery of identifiable fingermarks. This might be achieved through new and improved methods, but it will be dependent on having a better understanding of latent fingermarks and the interactions between secretions and different substrates. 


\section{Limitations of study}

525 When undertaking a study such as this, it is important to consider the limitations that may 526 affect the interpretation of the results. The biggest limitation was the number of donors 527 chosen for analysis (four males and one female). Six more donors were sampled for this study 528 but were removed from the analysis presented in this paper due to the low number of repeat 529 depositions collected. A decision was made to focus on the five donors who had provided the 530 greatest number of specimens to gain a better understanding of the repeatability of individual 531 donor characteristics. The other limitation was the fingermark ageing periods considered, 532 with only three- and seven-day-old fingermarks being developed. Previous research has 533 indicated that fingermark age can have a significant effect on the quality and number of 534 recovered marks, particularly for non-porous substrates. However, since the focus of this 535 particular project was on donor and detection factors, fingermark age was not considered as a 536 major test variable in this study. An avenue for future work would be to increase the aging period to understand the trends across a longer period of time.

\section{Conclusions}

541 Recent developments in fingermark detection research have focused on the application of 542 new technologies to increase the number and quality of fingermarks detected. While this

543 research is important to provide fingermark examiners with more efficient techniques, there 544 continues to be a lack of understanding of the actual effectiveness of routinely used 545 techniques. This study focused on understanding the factors that affect fingermark 546 development quality on a large pool of naturally deposited fingermarks. Through this study, it 547 has been demonstrated that fingermark detection quality is dependent on a variety of factors 548 that researchers should be aware of in order to increase the reliability of their results. In 549 particular, fingermarks deposited by the same donor display significant differences in quality, 550 even when other factors (i.e., fingermark age, substrate, detection technique) are controlled. 551 This finding should raise awareness that a large number of fingermarks (either in in the form 552 of a large donor pool and/or a large number of marks per donors) is required for more meaningful results. While there have been positive steps in standardising fingermark research

554 [11], there is still a need to improve our research design and reporting of fingermark detection 
555 success rates. A review of how we assess relative development quality should also be 556 conducted as current methods can give misleading results if deposition pressure and 557 distortion are not controlled (as these can impact on an assessment score independent of the 558 detection method). Ultimately, this study reaffirms the need for ongoing fingermark detection 559 research in order to increase success rates in operational casework.

560 
[1] N. Egli, S. Moret, A. Bécue, C. Champod, Fingermarks and other impressions, 17th

Interpol International Forensic Science Managers Symposium, Lyon 8 th-10 th October 2013, 2013, p. 745. [2] A. Bécue, C. Champod, Fingermarks and other body impressions - A review (July 2013 July 2016), in: Interpol (Ed.) $18^{\text {th }}$ Interpol Forensic Science Symposium, Lyon, France, 2016. [3] Bécue A., Cantú A. A. (2012), Fingermark detection using nanoparticles, In: Lee and Gaensslen's advances in fingerprint technology, 3rd ed., Ramotowski, R. S., Ed., CRC Press LLC; pp 307-379.. nano-structured particles in fingermark detection, Forensic Science International 179(2)

573 (2008) 87-97.

[5] M. Tahtouh, J.R. Kalman, C. Roux, C. Lennard, B.J. Reedy, The detection and enhancement of latent fingermarks using infrared chemical imaging, Journal of Forensic Science 50(1) (2005) 64-72

[6] M. Wood, P. Maynard, X. Spindler, C. Roux, C. Lennard, Selective targeting of fingermarks using immunogenic techniques, Australian Journal of Forensic Sciences 45(2) (2013) 211-226.

[7] S. Moret, X. Spindler, C. Lennard, C. Roux, Microscopic examination of fingermark residues: opportunities for fundamental studies, Forensic Science International 255 (2015) 28-37.

[8] N. Jaber, A. Lesniewski, H. Gabizon, S. Shenawi, D. Mandler, J. Almog, Visualization of latent fingermarks by nanotechnology: reversed development on paper - a remedy to the variation in sweat composition, Angewandte Chemie International Edition 51(49) (2012) 12224-12227.

[9] S. Wiesner, E. Springer, Y. Sasson, J. Almog, Chemical development of latent fingerprints: 1, 2-indanedione has come of age, Journal of Forensic Science 46(5) (2001) 1082-1084.

[10] L. McMullen, A. Beaudoin, Application of Oil Red O Following DFO and Ninhydrin Sequential Treatment: Enhancing Latent Fingerprints on Dry, Porous Surfaces, Journal of Forensic Identification 63(4) (2013) 387-423.

593 [11] Interational Fingerprint Research Group, Guidelines for the assessment of fingermark 594 detection techniques, Journal Forensic Identification. 64(2) (2014) 174-200. [12] A. Boudreault, A. Beaudoin, Pseudo-Operational Study on the Efficiency of Various Fingermark Development Techniques During the Aging Process, Journal of Forensic Identification 67(1) (2017) 85-117.

[13] Wilkinson D., Rumsby D., Babin B., Merritt M., Marsh J. (2005), The results from a canadian national field trial comparing 1,8-diazafluoren-9-one (DFO) with ninhydrin and the sequence DFO followed by ninhydrin, Canadian Police Research Centre [14] P. Fritz, W. Van Bronswijk, E. Patton, S.W. Lewis, Variability in Visualization of Latent Fingermarks Developed with 1, 2-Indanedione-Zinc Chloride, Journal of Forensic Identification 63(6) (2013) 698-713. 
[16] M. Levin-Elad, Y. Liptz, K.L. Bar-Or, J. Almog, 1,2-Indanedione - A winning ticket for developing fingermarks: A validation study, Forensic Science International 271(Supplement C) (2017) 8-12.

[17] A.A. Frick, P. Fritz, W. van Bronswijk, A. Beaudoin, S. Bleay, C. Lennard, S.W. Lewis, Investigations into the Influence of Donor Traits on Performance of Firigermark Development Reagents. Part 2: Oil Red O and PD, Journal of Forensic Identification 67(3) (2017) 427-446.

[18] N.N. Daéid, S. Carter, K. Laing, Comparison of vacuum metal deposition and powder suspension for recovery of fingerprints on wetted nonporous surfaces, Journal of Forensic Identification 58(5) (2008) 600-613. [19] A. Khuu, S. Chadwick, X. Spindler, R. Lam, S. Moret, C. Roux, Evaluation of one-step luminescent cyanoacrylate fuming, Forensic Science International 263 (2016) 126-131. [20] H. Earwaker, R.M. Morgan, A.J. Harris, L.J. Hall, Fingermark submission decisionmaking within a UK fingerprint laboratory: Do experts get the marks that they need?, Science \& Justice 55(4) (2015) 239-247.

[21] S. Fieldhouse, Consistency and reproducibility in fingermark deposition, Forensic Science International 207(1-3) (2011) 96-100.

[22] O.P. Jasuja, M. Toofany, G. Singh, G. Sodhi, Dynamics of latent fingerprints: The effect of physical factors on quality of ninhydrin developed prints-A preliminary study, Science \& Justice 49(1) (2009) 8-11.

[23] H. Reed, A. Stanton, J. Wheat, J. Kelley, L. Davis, W. Rao, A. Smith, D. Owen, S. Francese, The Reed-Stanton press rig for the generation of reproducible fingermarks: Towards a standardised methodology for fingermark research, Science \& Justice 56(1) (2016) 9-17.

[24] Champod C., Lennard C., Margot P., Stoilovic M. (2016), Fingerprints and other ridge skin impressions, second edition, CRC Press: Boca Raton, FL.

[25] V. Sears, S. Bleay, H. Bandey, V. Bowman, A methodology for finger mark research, Science \& Justice 52(3) (2012) 145-160.

[26] S.R. Bacon, J.J. Ojeda, R. Downham, V.G. Sears, B.J. Jones, The effects of polymer pigmentation on fingermark development techniques, Journal of Forensic Sciences 58(6) (2013) 1486-1494.

[27] K.T. Popov, V. Sears, B.J. Jones, Migration of latent fingermarks on non-porous surfaces: Observation technique and nanoscale variations, Forensic Science International 275 (2017) 44-56.

[28] D. E. Bicknell, R. Ramotowski, Use of an optimized 1,2-indandione process for the development of latent prints, Journal of Forensic Sciences 53(5) (2008) 1108-1116.

[29] T. G. Newland, S. Moret, A. Bécue, S. W. Lewis, Further investigations into the single metal deposition (SMD II) technique for the detection of latent fingermarks, Forensic Science International 268 (2016) 62-72.

[30] J.D. Wilson A.A. Cantu, G. Antonopoulos, M.J. Surrency Examination of the steps leading up to the physical developer process for developing fingerprints. Journal of Forensic Sciences, 52 (2007) 320-329

[31] A. Girod, C. Weyermann, Lipid composition of fingermark residue and donor classification using GC/MS, Forensic Science International 238 (2014) 68-82.

[32] R.S. Croxton, M.G. Baron, D. Butler, T. Kent, V.G. Sears, Development of a GC-MS Method for the Simultaneous Analysis of Latent Fingerprint Components, Journal of Forensic Sciences 51(6) (2006) 1329-1333. 
The authors are grateful and appreciative for the comprehensive feedback from both reviewers. Below are the responses to their questions, comments and suggestions. All minor grammatical, typographical or image corrections have been accepted and corrected in the manuscript.

Reviewer \#1: Overall, the authors present data obtained from a large scale study of the quality of latent print development on porous and non-porous surfaces. The data highlights the high degree of variability present in deposited print. I would recommend accepting this manuscript after the authors address some minor comments/corrections.

In the Abstract, what is meant by the authors when they say "finger on fingermark development"? Does this mean overlapping prints?

Authors Response: This statement was meant to refer the actual finger (thumb, index, ring etc) added 'type of finger' (Page 1 Line 15) to clarify this point.

In the Introduction, lines 87-98, have the authors also taken into consideration the impact of dark adaptation (or lack thereof) in a typical scenario when faint fluorescent prints are being viewed?

Authors Response: A fair point and is actually some of the future work being conducted based off this project. More specifically this future work aims to look at the threshold for the naked eye in visualising weak fingermarks and correlating that to conditions on the Poliview. No changes were made to the manuscript because in this specific study there was no dark adaptation due to the set up used in the laboratory and the paragraph in question focuses more on the deposition of fingermarks rather than the visualisation of them.

In the Introduction, page 5, line 6, the concept of "identification" is a very fluid one throughout the world. For example, most European countries have a range of point standards embedded in their legal systems. Others have no point standard. Since there is no consensus on what constitutes an identification, the use of some valuation scales can artificially lead to a decrease in efficiency for a technique. In most cases, if $1 / 3$ of a print is visible (levels $2-4$ on the CAST scale), it will most likely have enough ridge detail to be identifiable. This does not even take into consideration the fact that different formulations of the same reagent may be used (which may not all be equally effective).

Authors Response: Yes, we agree with this statement and this reinforces the challenges associated with assessing fingermark development quality. We have tried to be consistent and clear in regards to our definition of identification for out study but it is an issue when interpreting other studies and the number of identifiable marks recovered

On page 8 , line 97 , have the authors thought of future work that would compare trained/certified fingerprint examiners and researchers to evaluate the prints to see what differences might be detected?

Author Response: Yes this is certainly an avenue for us to explore, we hope that this work will generate some interest amongst fingerprint examiners so we can collaborate with them on future projects. No changes were made in the manuscript to that effect.

Page 8 , lines 103-107, see my previous comment that some prints given a score of 2 would be identifiable.

Authors Response: This is taken as a comment and no changes were made in the manuscript. 
On page 9, lines 134-137, why did the authors decide to originally age the prints for 3 and 7 days (which they admit should have been aged for longer periods)?

Authors Response: The primary laboratory work was completed by a research associate who was here for a total of 6 months. In order to maximise the number of marks that could be analysed shorter ageing periods were chosen to allow for enough time for the marks to be developed and imaged. This point is taken as a comment and no changes were made in the manuscript.

On page 10, line 151, while it would have significantly increased the complexity of this current study, the use of a sequence of development techniques instead of as single process would have improved recovery rates and one could have obtained valuable data on how each part of the sequence contributed to the overall success rate for development (there is a critical lack of data of this type available). Did the authors consider this during the planning of this or future experiments?

Authors Response: A previous study by Marriot et al performed a very thorough evaluation of different fingermark sequences on porous surfaces. However given the results of this work it would be valuable to look at the change in fingermark quality throughout the sequence looking at the factors examined in this study.

Page 21, it would seem to this reviewer that based on the authors' data and discussions that the currently available evaluation scales for developed fingerprints are not adequate. Based on the large quantity of data that the authors produced in this study, do they have any recommendations on how to modify the existing scales to more accurately reflect the data obtained? For example, very intense, but smudged, prints could be classified in a separate category to avoid inflating scored using the CAST system. Also, the use of fingerprint experts could also eliminate this problem as they could make a determination of whether the smudged print is identifiable or not.

Authors Response: As authors we agree that the current scales have some shortcomings in regards to the ability to evaluate low quality fingermarks. It was found that in this study fingermarks could either be deemed unidentifiable due to the deposition of the mark causing smudges or 'overloading' of the ridges resulting in a loss of detail or the inability of the development technique in given enough contrast for the mark to be appropriately visualised.

The following was added to the manuscript (page 21, line 367) 'A recommendation could be made to amend the current evaluation methods to have a qualifier for poorly developed (unidentifiable) marks categorised into poor development due to deposition or due to the technique to provide clearer reporting when assessing the ability of a technique to develop fingermarks. ' 